1. De la Cruz I, Reyes N, Rojas JL. Neumomediastino espontáneo en un consumidor de cocaína. Arch Bronconeumol 2000; 36: 231.

2. Maravelli A, Skiendzielewski JJ. Snover W. Pneumomediastinum acquired by glass blowing. J Emerg Med 2000; 19: 145-147.

3. Crespo D, Iglesias C, Márquez L, Panadero E, Vázquez P. Neumomediastino espontáneo idiopático: a propósito de un caso. An Pediatr 2006; 64: 106-107.

4. Santiago IJ, Martínez-Bayarri M. Neumomediastino espontáneo. Análisis de 16 casos. Emergencias 2000; 12: 321-325.

5. Campillo A, Coll A, Soria V, et al. Neumomediastino espontáneo. Estudio descriptivo de nuestra experiencia con 36 casos. Arch Bronconeumol 2005; 41: 528-31.

\section{Epiploitis aguda: una causa inusual de abdomen agudo médico}

\section{Sr. Director:}

La epiploitis aguda es una causa benigna, autolimitada y poco frecuente de abdomen agudo, que requiere un alto índice de sospecha; ya que puede simular múltiples entidades responsables de las principales causas de abdomen agudo quirúrgico y conducir de éste modo a una intervención innecesaria. Presentamos un caso de epiploitis aguda de evolución favorable con tratamiento conservador.

Varón de 29 años con antecedentes de apendicectomía y taquicardia paroxística supraventricular que consultó por presentar desde hacía 6 horas cuadro de dolor abdominal agudo localizado en fosa ilíaca izquierda (FII). A la exploración destacaba una $\mathrm{T}^{\mathrm{a}}$ de $37^{\circ} \mathrm{C}$ y peritonismo localizado en FII, siendo el resto normal. La analítica de sangre, Rx abdomen y ecografía abdominal fueron normales por lo que se pautó paracetamol (500 mg/8horas). Tras cuatro días de tratamiento consultó de nuevo por persistencia de la clínica. A la exploración persistía peritonismo localizado en FII, por lo que se realizó TC abdominal que se informó como aumento de densidad de la grasa en fosa ilíaca izquierda inmediatamente por debajo del peritoneo sugestivo de epiploitis (Fig. 1). Ante dicho diagnóstico se inició tratamiento antiinflamatorio: Ibuprofeno $(600 \mathrm{mg} / 8$ horas $)$ desapareciendo progresivamente la sintomatología en dos semanas.

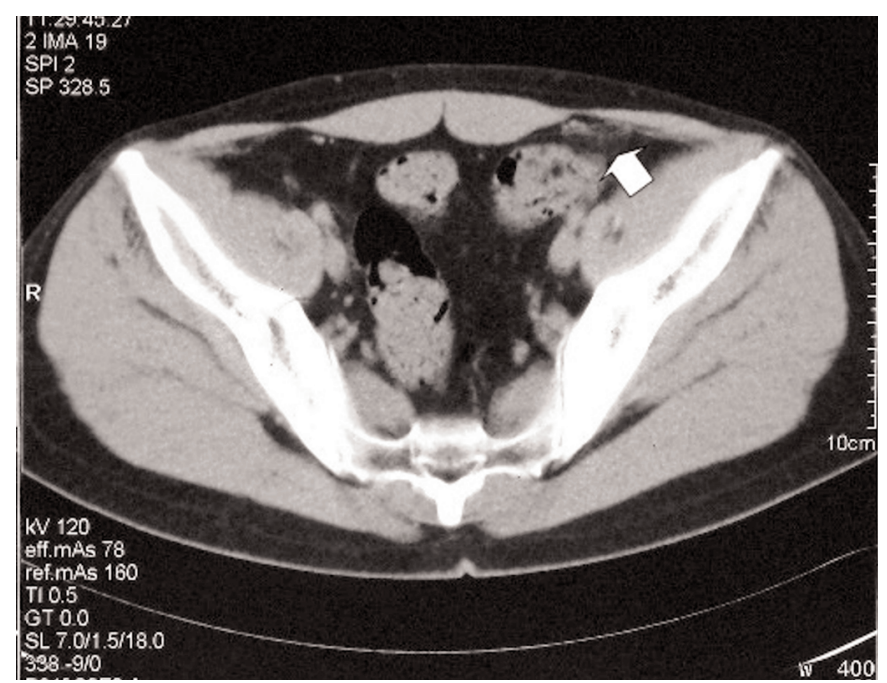

Fig. 1. TC abdominal: aumento de densidad de la grasa en FII.
La epiploitis aguda o apendicitis epiplóica, es una patología benigna y autolimitada, secundaria a la torsión o trombosis espontánea de las venas de drenaje de los apéndices epiploicos que puede similar un abdomen agudo quirúrgico. Ocurre habitualmente entre la segunda y quinta décadas, con una incidencia similar entre hombres y mujeres $(1,2)$. Los apéndices epiploicos, mencionados la primera vez por Vesalius en 1.543, son pequeños sacos constituidos por la duplicación del peritoneo visceral que rodean el intestino grueso entre el ciego y la unión recto-sigmoidea (3). Existen de 100 a 150 , y su longitud varía entre 0,5 y $5 \mathrm{~cm}$., conteniendo únicamente tejido adiposo y estructuras vasculares. La vascularización es terminal y la extrema movilidad de estas pequeñas estructuras pediculadas hacen posible su torsión, que representa su patología más frecuente. Como consecuencia, se produce un infarto isquémico de la grasa con irritación peritoneal, ocasionando un abdomen agudo, que en ocasiones simula un cuadro quirúrgico. Como factores predisponentes se han descrito la obesidad, la pérdida excesiva de peso o la existencia de hernias. Clínicamente se caracteriza por la presencia de dolor abdominal intenso, permanente y muy localizado. La localización es variable; sin embargo predomina en fosa ilíaca izquierda, donde los apéndices epiploicos son de mayor número y tamaño, o la fosa ilíaca derecha. En general no hay defensa abdominal; aunque sí existe blumberg localizado, palpándose en ocasiones una masa subparietal. Otros síntomas como alteración del tránsito intestinal, náuseas o vómitos, son raros. No suele existir fiebre, leucocitosis ni aumento significativo de la VSG (4,5). Respecto a las técnicas de imagen, la radiografía simple carece de utilidad. La ecografía puede mostrar una masa hiperecogénica, ovalada, en contacto con el colon y adherida a la pared abdominal, que mide de 1,5 a $5 \mathrm{~cm}$. de diámetro. La hiperecogenicidad se explica por el carácter adiposo del apéndice epiploico y por la necrosis hemorrágica que se produce en la grasa. Un fino halo hipoecogénico la rodea en más de la mitad de las lesiones, representando la serosa inflamada recubierta por un exudado fibroleucocitario (6). La TAC es el método de elección: muestra una masa hipodensa ubicada entre la serosa del colon y el peritoneo parietal, limitada por un anillo denso de 1 a $2 \mathrm{~mm}$ de grosor, que corresponde al halo hipoecogénico descrito en la ecografía. Ocasionalmente se visualizan algunas finas líneas densas en el centro de la lesión, que representan los vasos trombosados del apéndice epiploico. La infiltración de la grasa circundante es constante y se traduce por el aumento de la densidad de ésta. Otro fenómeno muy frecuente (90-98\%) es el engrosamiento del peritoneo parietal en la zona vecina. Estos hallazgos pueden persistir hasta 6 meses después de haber presentado el cuadro. Asimismo la TAC es útil para descartar el resto de patologías con las que se debe realizar el diagnóstico diferencial; incluyendo entre otras, la diverticulitis aguda, apendicitis, paniculitis mesentérica, infarto omental, tumores/metástasis en mesocólon o traumatismos. Existen series, en las que se observó que entre el 2-7\% de pacientes con sospecha de diverticulitis, y entre el $0,3-1 \%$ de sospechas de apendicitis fueron realmente epiploitis $(7,8)$. El tratamiento es conservador, empleando antiinflamatorios o opiáceos durante 4-7 días; con lo que habitualmente se resuelve el cuadro en menos de 2 semanas. Las complicaciones son raras, aunque ocasionalmente el material inflamado se puede adherir a las vísceras ocasionando obstrucción intestinal (9).

En conclusión, ante un abdomen agudo localizado, especialmente en paciente jóven, afebril y sin alteraciones analíticas relevantes se debe incluir la epiploitis aguda en el diagnóstico diferencial, para evitar de este modo hospitalizaciones, terapia antibiótica o intervenciones quirúrgicas innecesarias.

\section{Teijo Núñez, E. Raimunde Seoane, S. Álvarez Álvarez, J. L. Olcoz Goñi}


1. Van Breda Vriesman AC, Lohle PN, Coerkamp EG, Puylaert JB. Infarction of omentum and epiploic appendage: diagnosis, epidemiology and natural history. Eur Radiol 1999; 9: 1886-1892.

2. Legome EL, Belton AL, Murray RE, Rao PM, Novelline RA. Epiploic appendagitis: the emergency department presentation. J Emerg Med 2002; 22: 9.

3. Feber SS, Forman J. Appendices epiploicae: clinical and pathological considerations; report of 3 cases and statistical analysis on 105 cases. AMA Arch Surg 1953; 66: 329-338.

4. Son HJ, Lee SJ, Lee JH, Kim JS, Kim YH, et al. Clinical diagnosis of primary epiploic appendagitis: differentiation from acute diverticulitis. J Clin Gastroenterol 2002; 34: 435-438.

5. Rao PM, Rhea JT, Novelline RA, Mostafavi AA, McCabe CJ. Effect of computed tomography of the appendix on treatment of patients and use of hospital resources. N Engl J Med 1998; 338: 141.

6. Rioux M, Langis P. Primary epiploic appendagitis: clinical, US, and CT findings in 14 cases. Radiology 1994; 191: 523-526.

7. Singh AK, Gervais DA, Hahn PF, Sagar P, Mueller PR, et al. Acute epiploic appendagitis and its mimics. Radiographics 2005; 25: 1521-1534.

8. Sandrasegaran K, Maglinte DD, Rajesh A, Akisik FM. Primary epiploic appendagitis: CT diagnosis. Emerg Radiol 2004; 11: 9-14.

9. Puppala AR, Mustafa SG, Moorman RH, Howard CH. Small bowel obstruction due to disease of epiploic appendage. Am J Gastroenterol 1981; 75: 382 .

\section{Prevalencia de anemia en pacientes con insuficiencia cardiaca}

\section{Sr. Director:}

La anemia tiene una elevada prevalencia (15-30\%) en los pacientes con insuficiencia cardiaca $(1,2)$, incrementándose según la gravedad de la misma, del $9 \%$ para la clase I de la NYHA, hasta el 79\% en la clase IV (3).

La insuficiencia cardiaca congestiva puede contribuir al desarrollo de la anemia y a su vez la anemia en el paciente que tiene una insuficiencia cardiaca constituye un factor de mal pronóstico, independiente para la mortalidad $(4,5)$.

La anemia de la insuficiencia cardiaca congestiva tiene un origen multifactorial y puede deberse $\mathrm{a}: \mathrm{a}$ ) déficit de hierro por disminución del aporte, malabsorción o pérdidas crónicas; b) disminución de la producción de eritropoyetina (EPO) por la insuficiencia renal crónica, que con elevada frecuencia presentan estos enfermos y también por la toma de fármacos de la familia de los inhibidores de la enzima de conversión de la angiotensina (IECA); c) pérdida urinaria de eritropoyetina y de transferrina por la proteinuria; d) inhibición de la eritropoyesis, por el factor de necrosis tumoral alfa que se encuentra elevado en estos pacientes, y e) hemodilucción, por el incremento del volumen plasmático (6).

Un déficit funcional de hierro, típico de la enfermedad crónica, puede desempeñar un papel importante en la anemia de los pacientes con insuficiencia cardiaca.

El tratamiento de la anemia en los pacientes con insuficiencia cardiaca, básicamente con hierro y EPO a dosis bajas, tiene una efectividad bien documentada (6).

Presentamos los resultados de un estudio en el que analizábamos la prevalencia de anemia en un grupo de pacientes diagnosticados de insuficiencia cardiaca. Excluimos del estudio los pacientes diagnosticados de insuficiencia renal crónica, hernia de hiato ó ulcus péptico gastroduodenal, procesos hematológicos o oncohematológicos, diversos cuadros reumatológicos crónicos que cursan frecuentemente con anemia y pacientes diagnosticados de anemia por cualquier otra causa.

También excluimos a los enfermos con enfermedad pulmonar obstructiva crónica (EPOC), dado que pueden presentar poliglobulia, que constituiría un factor de confusión en el estudio.

Realizamos un estudio retrospectivo, observacional y de prevalencia

Se incluyeron los enfermos ingresados por insuficiencia cardiaca como diagnóstico principal, en un servicio de Medicina Interna de un hospital del grupo 2, en el plazo de 9 meses, excluyendo los pacientes con los diagnósticos previos de exclusión referidos anteriormente.

Finalmente el grupo de estudio estuvo constituido por 46 pacientes, 26 mujeres y 20 varones con edades comprendidas entre 61 y 97 años, con una edad media de 78,8 años.

El valor medio de la hemoglobina fue de $12,9 \mathrm{~g} / \mathrm{dl}$.

Tenían anemia, definida como un valor de hemoglobina inferior a $12 \mathrm{~g} / \mathrm{dl}$, en las mujeres y de $13 \mathrm{~g} / \mathrm{dl}$ en los varones, un total de 14 pacientes $(30,4 \%)$.

Unicamente cuatro pacientes tenían un valor medio de hemoglobina inferior a 10 gramos/dl $(8,6 \%)$.

La edad media de estos 14 pacientes era de 75,8 años, por lo tanto ligeramente inferior a la global del grupo, pero sin diferencias relevantes.

No podemos precisar por no constar en el informe de alta en todos los casos, la clasificación de la gravedad de la insuficiencia cardiaca, aunque teniendo en cuenta la avanzada edad de los pacientes y los casos en los que estaba consignada (18 pacientes, 14 grado III y 4 grado IV), una gran mayoría de los mismos estaba encuadrado en los grupos III y IV de la NYHA.

Resulta llamativo la elevada proporción de pacientes con anemia que encontramos en nuestro estudio (30\%), aún más teniendo en cuenta el elevado número de pacientes que excluimos por presentar procesos que podrían justificar la anemia.

La mayoría de los pacientes con anemia fueron remitidos para estudio y seguimiento posterior en la consulta externa de Medicina Interna.

Como conclusiones podemos decir que la anemia es muy frecuente en los pacientes ancianos con insuficiencia cardiaca y aumenta con la gravedad de la enfermedad.

\section{F. Marcos Sánchez, M. I. Albo Castaño, D. Joya Seijo, P. del Valle Loarte, S. Casallo Blanco}

Servicio de Medicina Interna. Hospital Nuestra Señora del Prado. Talavera de la Reina. Toledo

1. Urrutia A, Lupón J, González B, Parajón T, Altimir S, Coll R, et al. Prevalencia de la anemia y su correlación con los parámetros clínicos en pacientes de una unidad multidisciplinaria de insuficiencia cardiaca. Med Clin (Barc) 2004; 122: 121-125.

2. Tanner H, Moschovitis GM, Hullin R, Pfiffner D, Hess OM, Mohacsi P The prevalence of anemia in chronic heart failure. Int J Cardiol 2002; 86: 115-121.

3. Silverberg DS, Wesler D, Blum M, Keren G, Sheps D, Leibovitch E, et al. The use of subcutaneous erythropoietin and intravenous iron for the treatment of anemia of severe, resistant congestive heart failure improves cardiac and renal function and functional cardiac class, and markedly reduces hospitalisations. J Am Coll Cardiol 2000; 35: 1737-1744.

4. Ezekowitz JA, McAliste FA, Amstrong PW. Anemia is common in heart failure and is associated with poor outcomes. Circulation 2003; 107: 223 225.

5. Sharma R, Francis DP, Pitt B, Poole-Wilson PA, Coats AJ, Anker SD. Haemoglobin predicts survival in patients with chronic heart failure: a substudy of the ELITE II trial. Eur Heart J 2004; 25: 1021-1028.

6. Silverberg DS, Wesler D, Blum M, Laina A. The importance of anemia and its correction in the management of severe congestive heart failure. Eur J Heart Failure 2002; 4: 681-686. 